INPLASY

PROTOCOL

To cite: Zhao et al. Evening dosing versus morning regimen drug therapy for hypertension: a meta-analysis of randomized controlled trial. Inplasy protocol 202180005. doi:

10.37766/inplasy2021.8.0005

Received: 02 August 2021

Published: 02 August 2021

Corresponding author: Jing Zhao

zhaojingyisheng@163.com

Author Affiliation:

Dongzhimen Hospital, Beijing University of Chinese

Medicine.

Support: NKRDP of China.

Review Stage at time of this submission: Preliminary searches.

Conflicts of interest: None declared.

\section{Evening dosing versus morning regimen drug therapy for hypertension: a meta-analysis of randomized controlled trial}

Zhao, J1; Zeng, Y2; Weng, J3; Zhang, J4; Yao, T5; Yuan, M6; Shen, $X^{7}$.

Review question / Objective: P:hypertension patients; l:evening dosing regimen drug therapy; C:morning dosing regimen drug therapy; O:cardiovascular diseases events, diurnal mean of systolic blood pressure (SBP)/ diastolic blood pressure (DBP), nocturnal mean of SBP/DBP, and 24h/48h mean of SBP/DBP by ABPM; S:randomized controlled trial. Eligibility criteria: (1) The study was a randomized controlled trial (RCT); (2) Adult patients with primary (essential) hypertension or secondary causes of hypertension whose systolic and/or diastolic blood pressure levels were 140/90 $\mathrm{mmHg}$ or greater were included. Patients with white coat hypertension and alternating shift workers were excluded; (3) Intervention was defined as one or more antihypertensive drugs administered once-daily in the evening (from 6:00 p.m. to 12:00 midnight), the control group was defined as one or more antihypertensive drugs administered once-daily in the morning (from 6:00 a.m. to 12:00 noon). Antihypertensive drug belonging to any one of the following six classes: ACEIs, ARBs, CCBs, beta-blockers ( $\beta$ - blockers), alpha-blockers ( $\alpha$ blockers) and diuretics. (4) Outcomes were cardiovascular diseases events, diurnal mean of systolic blood pressure (SBP)/ diastolic blood pressure (DBP), nocturnal mean of SBP/ DBP, and 24h/48h mean of SBP/DBP by ABPM.

INPLASY registration number: This protocol was registered with the International Platform of Registered Systematic Review and Meta-Analysis Protocols (INPLASY) on 02 August 2021 and was last updated on 02 August 2021 (registration number INPLASY202180005).

\section{INTRODUCTION}

Review question / Objective: P:hypertension patients; l:evening dosing regimen drug therapy; C:morning dosing regimen drug therapy; O:cardiovascular diseases events, diurnal mean of systolic blood pressure (SBP)/ diastolic blood pressure (DBP), nocturnal mean of SBP/ 
DBP, and $24 \mathrm{~h} / 48 \mathrm{~h}$ mean of SBP/DBP by ABPM; S:randomized controlled trial.

Condition being studied: Hypertension is a global public health crisis affecting an estimated 1.13 billion around the world and 4.5 million in China. It is a major cause of premature death worldwide, which could increase the risk of heart, brain, kidney and other diseases as a serious medical condition. Both ESC/ESH and ACC/AHA guidelines for the management of arterial hypertension suggesting that most patients will require drug therapy in addition to lifestyle measures to achieve optimal blood pressure control, with five major drug classes were recommended for the treatment of hypertension: angiotensinconverting enzyme inhibitors (ACEIs), angiotensin receptor blockers (ARBs), beta-blockers( $\beta$-blockers), calcium channel blockers (CCBs) and diuretics (thiazides and thiazide-like).The benefits of blood pressure lowering treatment could reduce cardiovascular, renal events and total death risk. The ambulatory blood pressure monitoring (ABPM) provides a larger number of blood pressure measurements than conventional office blood pressure in conditions that are more representative of daily life. The MAPEC study demonstrated that bedtime therapy relative to conventional morning therapy better reduces the asleep blood pressure mean, with additional benefit, that is $61 \%$ reduction of cardiovascular disease events. However, there is still a lack of relevant clinical evidence. Therefore, we conducted this meta-analysis to investigate the effects of evening administration of blood pressure on ABPM results and cardiovascular disease events.

\section{METHODS}

Participant or population: Hypertension patients.

Intervention: Evening dosing regimen drug therapy.

Comparator: Morning dosing regimen drug therapy.
Study designs to be included: Randomized controlled trial.

Eligibility criteria: (1) The study was a randomized controlled trial (RCT); (2) Adult patients with primary (essential) hypertension or secondary causes of hypertension whose systolic and/or diastolic blood pressure levels were 140/90 $\mathrm{mmHg}$ or greater were included. Patients with white coat hypertension and alternating shift workers were excluded; (3) Intervention was defined as one or more antihypertensive drugs administered oncedaily in the evening (from 6:00 p.m. to 12:00 midnight), the control group was defined as one or more antihypertensive drugs administered once-daily in the morning (from 6:00 a.m. to 12:00 noon). Antihypertensive drug belonging to any one of the following six classes: ACEls, ARBs, CCBs, beta-blockers ( $\beta$ - blockers), alphablockers ( $\alpha$ - blockers) and diuretics. (4) Outcomes were cardiovascular diseases events, diurnal mean of systolic blood pressure (SBP)/ diastolic blood pressure (DBP), nocturnal mean of SBP/DBP, and $24 \mathrm{~h} / 48 \mathrm{~h}$ mean of SBP/DBP by ABPM.

Information sources: Databases including PubMed, The Cochrane Library, EMbase, Web of Science, CBM, WanFang Date, VIP and CNKI database.

Main outcome(s): Cardiovascular diseases events.

Quality assessment / Risk of bias analysis: Two independent reviewers assessed the risk of bias of all included trials and completed a Risk of Bias Table as described in the Cochrane Handbook.

Strategy of data synthesis: Two investigators extracted the following data independently from each of the studies includes: the first author; year of publication; study country; funding support; patient characteristics (age, sex ratio, hypertension type); interventions (grouping, sample size, types of drugs, intervention duration); outcomes. In the case of missing information in the included studies, investigators were contacted by email to 
obtain the missing information.Metaanalysis was conducted using Stata 12.0 software. Heterogeneity between trial results was tested using the $Q$ test and the I2 statistic where percentages greater than $50 \%$ were taken to indicate significant heterogeneity. If heterogeneity was detected for outcomes, meta regression, subgroup analysis and sensitivity analysis were performed to analyze the causes of heterogeneity. The test level of meta regression was set as $a=0.1$. Funnel plot and Begg test were used to evaluate the publication bias. If the results indicated that the possibility of bias was high, the stability of the research results was analyzed by sensitivity analysis. The test level of meta-analysis was set as $\mathbf{a}=\mathbf{0 . 0 5}$.

Subgroup analysis: Grouping, sample size, types of drugs and intervention duration.

Sensitivity analysis: If the results indicated that the possibility of bias was high, the stability of the research results was analyzed by sensitivity analysis.

Country(ies) involved: China.

Keywords: Hypertension; Blood pressure; Evening administration; Meta-analysis; Randomized controlled trial.

Contributions of each author:

Author 1 - Jing Zhao.

Author 2 - Yuanyuan Zeng.

Author 3 - Jieqiong Weng.

Author 4 - Jingfang Zhang.

Author 5 - Tingting Yao.

Author 6 - Mengfei Yuan.

Author 7 - Xiaoxu Shen. 